\title{
Research on Configurable Workflow Technology in PDM System
}

\author{
Kang CAOa, Li LIU'b, and Zheng CHENG \\ Institute of Computer Application, China Academy of Engineering Physics, Mianyang Sichuan ,621900, China \\ ackhad@126.com, bliuli@caep.cn, ${ }^{\circ}$ cghz2804@163.com \\ Corresponding author: Li LIU
}

\begin{abstract}
This paper analyzed the deficiency of the static process management in the traditional PDM workflow system, and proposed a new configurable workflow system model for the dynamic and multiple characteristics of the equipment product design business process. On the basis of PDM workflow engine, the model introduced the configurable workflow form and logic processing program, the total workflow template definition and customized workflow participant selection module, and put forward the key technology solutions. This model could solve the problem of the lack of flexibility in workflow design and maintenance. It had been successfully applied to the design management process for multiple equipment products, and improved the dynamic process management capability of PDM system.
\end{abstract}

\section{Introduction}

Workflow is presented in order to improve the work efficiency. By decomposing the work activities into well-defined tasks, roles, rules and processes, the execution and monitoring of the workflow is achieved, so as to improve the level of the production organization and the efficiency of the work. Workflow Management Coalition defines workflow as: The whole or part of the business process automation.In the process, documents, information or tasks flow according to certain rules of the process, to achieve the coordination among the organization members and the overall objectives of the business.[1]

Based on product structure, with production data as the core, product data management (PDM) system uses modern network communication technology and database technology, realizes the integration management of design tools, design data, design process and design resources. Through logically connecting each CAX information isolated island, PDM can manage the whole product design and development process, gradually establish a virtual product model, and finally form a complete product description, production process description and production process control data. It provides a fine management and control mechanism for enterprise product related information and process. [2]

PDM usually has functions of product structure management, document management, report management and other static data management. At the same time, it also provides the function of process management to manage the dynamic flow of product data.

\section{Process Management in PDM System}

For the fixed business processes of similar business object, PDM workflow management system usually uses graphical process designer to define process tasks and execute logic, and completes process automatic execution by workflow engine. There may be many differences in the process management system of different PDM products, but they generally provide the following three aspects support: construction time function, run-time control function, run-time interactive function.

Construction time function. It is responsible for defining and organizing the business process, each task of process, and task control relationship. Process management module in PDM system generally provides the function of process template. Through the definition of process start and end, process task, task executor, executive order, authority of related data, etc. , we can construct a fixed workflow template in PDM system.

Run-time control function. It is responsible for creating, executing and controlling the workflow template, activating the corresponding resources and applications, controlling the transformation of a task to another task, using system message to notify task executor, and so on.

Run-time interactive function. It mainly refers to the interactive function between the workflow management system and the users (the workflow executor and the system administrator) and the external application program. Such as online browsing files, comments, route selection, etc.

According to the requirements of equipment product configuration management, the design documents and reports generated in the process of product design need to be audited and changed control. Because of the different 
organizational structure and management requirements, the examining and changing procedure of the product design files are different. In the complex equipment product design process, configuration management procedures of different type design files are different, even the same type design files also have differences in the different lifecycle stage. Therefore, for each type of business object constructing and executing a fixed workflow template by traditional PDM workflow management system, it will greatly increase the workload of the workflow definition and maintenance. That is difficult to adapt to the diversity and dynamic changes of product design process.

\section{Configurable Workflow System Model}

According to the configuration management requirements of complex equipment product design process, this paper established a configurable workflow system model based on the traditional PDM workflow system, to solve the problem of lack of flexibility in the design and implementation of the existing PDM workflow. As shown in Figure 1, the model includes the execution control layer, the logic processing layer and the user interface layer.
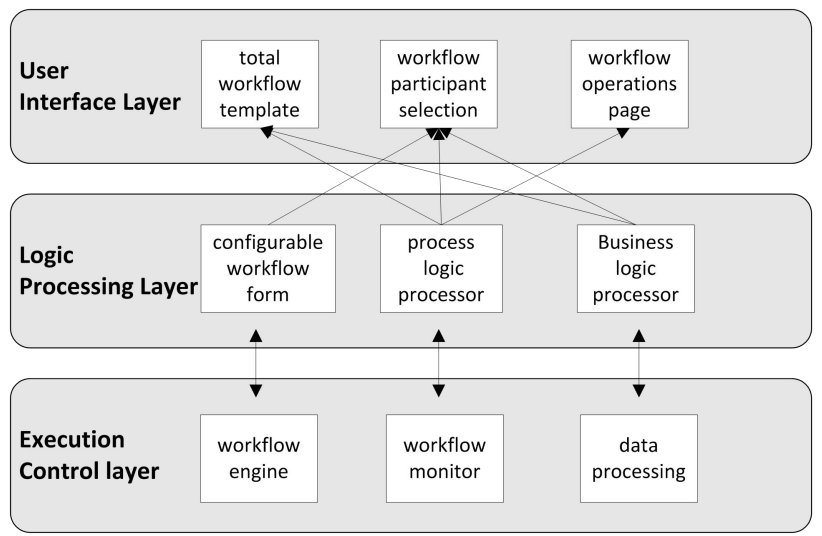

Figure 1. Configurable Workflow System Model

The execution control layer. It mainly realizes the execution of the workflow, the state monitoring and data processing. The workflow engine and interface API of PDM system are used to achieve the conversion, synchronization, asynchronous, cycle and other processing logic of the process node, to achieve process start, stop, recovery, monitoring and management of execution status and other operations. And it will complete data processing such as participant and organization structure storing in the database.

The logic processing layer. It provides business logic processing services for configurable workflow systems. According to the business requirements, configurable workflow form are designed. It can define the workflow of different business objects under different conditions; The total workflow of multi type business objects can be dynamically calculated by configurable workflow form which provides business logic support for the workflow participant selection; Combined with the workflow participant selection, the execution logic of the workflow node is dynamically determined through logic processing program. It support configurable workflow instance running.

The user interface layer. It provides graphical process definition and operation services for users. It uses the PDM workflow designer to define the total workflow template; For configuring the workflow runtime nodes, it inserts the workflow participant selection page into the workflow template, and associates the participant selection rules with the workflow configuration form by the business logic processing program; It uses PDM workflow system to realize the workflow task notification, workflow opinion recording and route selection, etc.

On the basis of traditional PDM workflow system , a configurable workflow system model is established by designing workflow configuration form and logic processing program, simplifying the method of workflow template definition and customizing workflow participant selection. In this model, configurable related content is mainly focused on the logic processing layer and user interface layer. The logic processing layer is the core of the configurable workflow, and the total workflow template definition and the workflow participant selection are the key of the configurable workflow implementation.

\section{Configurable Workflow Key Technologies}

\subsection{Total Workflow Template Definition}

On the basis of PDM workflow engine, the general total workflow template is defined according to the classification of business, rather than the traditional single workflow template for specific business. This workflow template is one of the largest sets of workflow nodes that may be involved in the process. It contains all the possible business conditions. Such as the design document approval process, the complete set of workflow template task node includes: proofread, audit, standardized examination, countersignature, technical approval and administrative approval. However, the design file does not need to countersignature, technical and administrative approval in the early stage of product design; Engineering drawings need countersignature by process department; Project report need countersignature by management department; Some files need countersignature by multiple departments. Thus, establishing a total workflow template for a class of business areas of enterprises and realizing the dynamic configuration of the process are conducive to the unified management and maintenance of business processes.

Workflow template uses a lot of workflow logical operations to organize and connect all the workflow task nodes. Such as conditional judgment branch, AND/OR connection, threshold value judgment, workflow wait and synchronization. It also uses the embedded code to call the executable program of the logic processing layer for complex logic processing, which will eventually build the complete workflow process. 


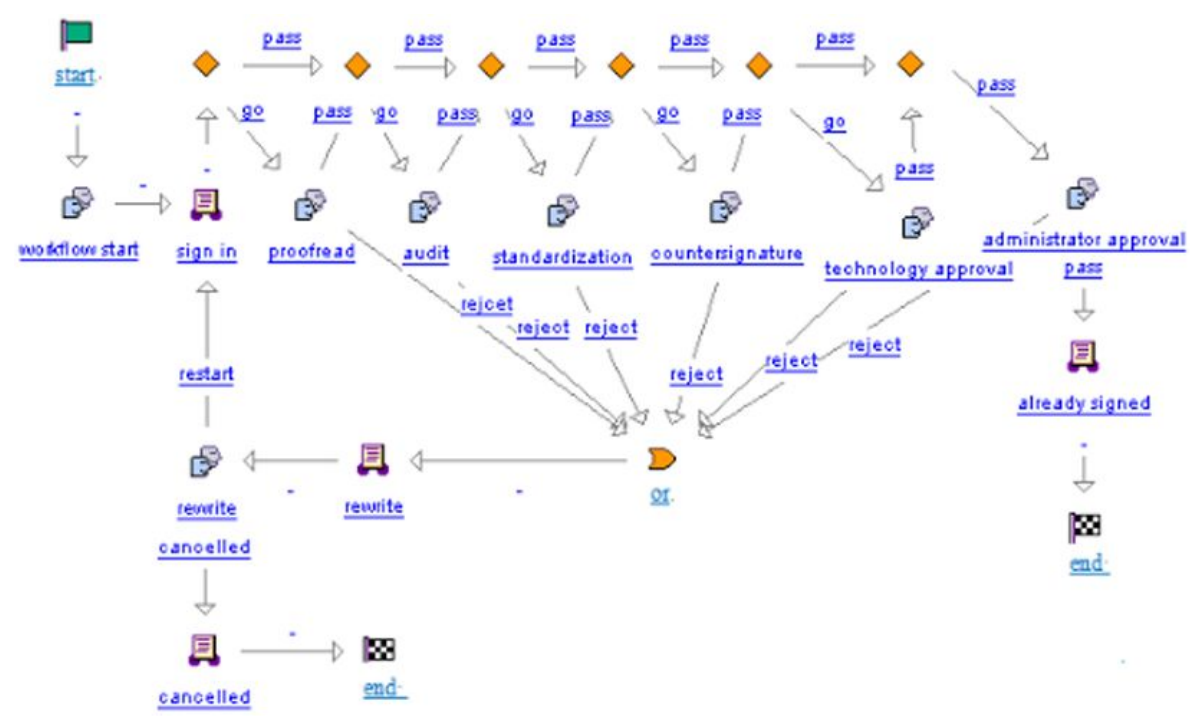

Figure 2. Example of Total Workflow Template

\subsection{Workflow Configuration form and Logic Processing Program}

On the basis of the total workflow template, in response to the specific requirements of product configuration management, the configurable workflow form is established. According to the business changes, business manager can dynamically adjust the workflow by this form. Workflow configuration form includes business objects, object attributes and process participants. It can realize the dynamic configuration of different business objects in different conditions. Configuration form can be applied to the full product design lifecycle of multiple products, single product, or product components. It works by a logic processing program when the workflow is running. Figure 3 is an example of document approval workflow configuration form. It defines the file types, process nodes, and design phases. It includes large, middle and small three kinds of document types, six document approval nodes, and five product design phases. It can configure process nodes of different file types in different design phases.For example, the assignment book in the document small class. It needs to proofread, audit, standardized examination, technical and administrative approval in all design phases, but countersignature only in the phase of G and D.

\begin{tabular}{|c|c|c|c|c|c|c|c|c|}
\hline $\begin{array}{l}\text { document } \\
\text { large class }\end{array}$ & $\begin{array}{l}\text { document } \\
\text { midđle class }\end{array}$ & $\begin{array}{l}\text { document } \\
\text { small class }\end{array}$ & prootread & audit & $\begin{array}{l}\text { standardized } \\
\text { exatmination }\end{array}$ & $\begin{array}{c}\text { counter } \\
\text { signsture }\end{array}$ & $\begin{array}{l}\text { technical } \\
\text { approval }\end{array}$ & $\begin{array}{c}\text { 2dministrative } \\
\text { spproval }\end{array}$ \\
\hline \multirow[t]{14}{*}{ Destgn Documents } & Writing class & assignment book & FCSGD & FCSGD & FCSGD & GD & FCSGD & FCSGD \\
\hline & & operating procedures & FCSGD & FCSGD & FCSGD & $G D$ & & GD \\
\hline & & specifications & FCSGD & FCSGD & FCSGD & $G D$ & & GD \\
\hline & & debugzing instructions & FCSGD & FCSGD & FCSGD & & & $G D$ \\
\hline & Report class & project report & FCSOD & FCSGD & FCSOD & $G D$ & FCSCD & FCSOD \\
\hline & & quahity analysis teport & FCSGD & FCSGD & FCSGD & $G D$ & & GD \\
\hline & & Research Report & FCSGD & FCSGD & FCSGD & GD & & GD \\
\hline & & opening report & FCSGD & FCSGD & FCSGD & & & GD \\
\hline & & originalrecording & FCSGD & FCSGD & FCSGD & & & $G D$ \\
\hline & & evaluation report & FCSGD & FCSGD & FCSCD & $O D$ & & $G D$ \\
\hline & & test report & FCSOD & FCSOD & FCSOD & $G D$ & & $G D$ \\
\hline & & process review report & FCSGD & FCSGD & FCSGD & GD & & GD \\
\hline & & component inspection report & FCSGD & FCSGD & FCSGD & $G D$ & & GD \\
\hline & & product inspection report & FCSGD & FCSGD & FCSGD & $G D$ & D & GD \\
\hline
\end{tabular}

Figure 3. Document Approval Process Configuration Form Example

The main processing logic of workflow configuration form is: When the process contains a single business object, according to the corresponding single record in the configuration form configure the workflow. When process contains more than one business object, reading more rows in the form through the corresponding object types, it will calculate the most complete workflow task nodes.

The core logic processing program code is as below:

if (obj instanceof WTDocument) // If the workflow object is a single document
WTDocument doc $=($ WTDocument $)$ obj;

String phase = IBAHelper.getIBAValue $($ doc, PartConstraints.IBA_YZJD);//get phase property value rtn $\quad$ getSignatureRoleDefine(qspz, TypedUtility.getTypeIdentifier(doc), phase);//get workflow nodes configuration for this file \}

else if (obj instanceof PromotionNotice) $\{/ /$ If the workflow object is a file package

QueryResult qr=MyPromotionHelpe.getPromotable(( PromotionNotice)obj);//access to all the objects in the 
file package

HashMap

sc.getAllDocumentTypeIdentifier();

Sheet sheet $=$ sc.getQspzSheet(qspz);//get the workflow configuration form

HashMap roleMap = sc.getAllRoleMap(sheet);//get configuration information about all workflow nodes in the configuration form

while (qr.hasMoreElements()) \{//get each object of file package

Persistable $\mathrm{p}=($ Persistable $)$ qr.nextElement () ;

if( $\mathrm{p}$ instanceof WTDocument) $\{/ /$ If it is a document

WTDocument doc $=($ WTDocument $) \mathrm{p}$;

String phase $=$ IBAHelper.getIBAValue(doc, PartConstraints.IBA_YZJD); // get phase property value TypeIdentifier ti

TypedUtility.getTypeIdentifier(doc);

ArrayList list $=$ sc.getSignatureRoleDefine(ti, phase,typeDef,sheet,roleMap);/get the workflow node configuration of this document type

rtn.addAll(list);//add to workflow nodes configuration information set

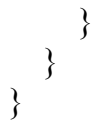

\subsection{Main Processing Logic of Configurable Workflow Instantiation Runtime}

The main processing logic of configurable workflow instantiation runtime is shown in figure 4 . The first step is to select workflow participants. According to process the business object and the configuration form, logical processing program summary calculate the necessary workflow nodes. They are displayed in a custom workflow participant selection page on the first workflow node. Workflow originator need to select participants for these necessary workflow nodes. All workflow personnel information will be stored in the database through the interface program. Before entering each workflow task node, workflow need to judge the existence of node. If the node has participants, it need to be performed. Otherwise it will go to the next node.

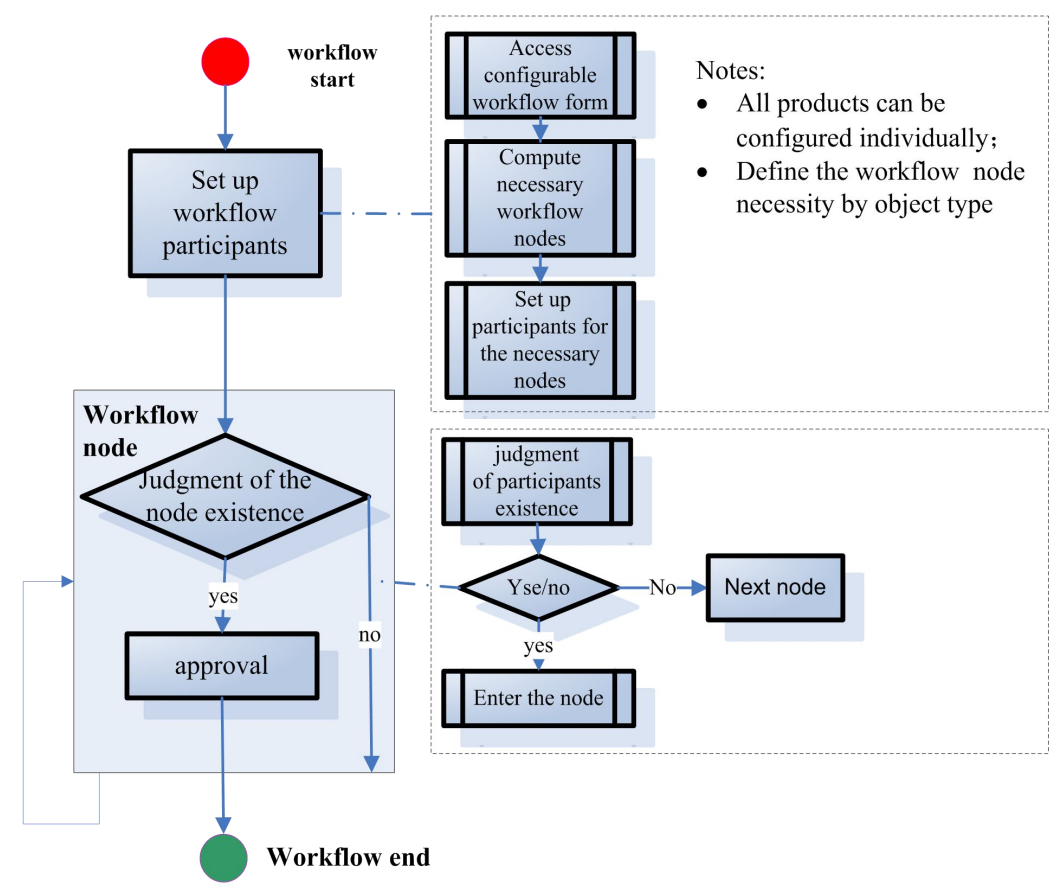

Figure 4. Main Processing Logic of the Configurable Workflow Runtime

The configurable workflow technology combines workflow task node selection and execution logic with workflow configuration form. Through summary calculation of the workflow task nodes, general workflow template is instantiated as a specific workflow that can satisfy the requirement of different configuration management. It implements the configurable workflow management in different products, different business objects and different conditions. The new workflow design pattern has been applied in the development and management of multiple equipment products. It reduced the workload of workflow template definition and process maintenance, simplified and facilitated the process redefinition and business adjustment for business manager.

\section{Summary}

In this paper, on the basis of analyzing the characteristics of the function of PDM workflow system and the requirements of equipment product configuration management, we proposed a configurable workflow system model, and realized total workflow template definition, workflow configuration form and logical processing program, the main processing logic of workflow template instantiation runtime, etc. It well solved the problem of the dynamics and diversity of 
product design process, and effectively improved the capacity of the process management in PDM system.

\section{References}

1. Y.S. Fan, Beijing: tsinghua university press (2001)

2. W.H. Fan, T. Li, Beijing: Mechanical Industry Press, 20 (2004)

3. Workflow Management Coalition Specification:Term Glossary, Document Number WfMC-TC-1011 (1996)

4. Y.P. Liu, W. Zhao, X. Xiong, Journal of Mechanical Design and Manufacturing 1, 225 (2009)

5. Z.H. Xu, Y.J. Dai, Y.F. Wang, Journal of Design and Research 5, 13 (2007)

6. Y.B. Guo, Z.Y. Cai, Z.W. Lin, Y.P. Peng, Journal of Computer Engineering and Design 3, 35 (2014)

7. W. Chao, Z.Z. Nong, W. Xiong, Journal of Computer Research and Development Suppl., 46 (2009) 